\title{
La "ruleta de moyers", instrumento auxiliar para el análisis de la dentición mixta basado en la ecuación de regresión lineal por mínimos cuadrados

\author{
"Moyers roulette", auxiliary instrument for the mixed dentition analysis based in the lineal \\ regression ecuation by minimal squares.
}

Héctor Marengo Castillo ${ }^{1 a}$, Yuri Castro-Rodríguez ${ }^{2 b}$, Sheyla Bravo Jaimes ${ }^{2 c}$, Geanny Calampa Sopla $^{2 c}$, Lizeth Torres Arévalo ${ }^{2 c}$

\section{RESUMEN}

Objetivo. El presente estudio tuvo como objetivo presentar un nuevo instrumento que facilite el análisis de Moyers en la dentición mixta, incluyendo valores desde los $19 \mathrm{~mm}$ hasta los 30,5 $\mathrm{mm}$ para la sumatoria de incisivos superiores e inferiores. métodos. Se utilizaron las tablas clásicas de Moyers y a través de cuatro ecuaciones lineales se proyectaron los datos tanto para valores de varones y mujeres; la ruleta fue construida con valores desde $19 \mathrm{~mm}$ hasta $30,5 \mathrm{~mm}$ correspondientes a las sumatoria de los cuatro incisivos inferiores. Se utilizó la regresión lineal y ecuaciones de primer grado para proyectar los valores tanto para el maxilar como para la mandíbula. El modelo de la ruleta fue ideado siguiendo el modelo del Ortómetro de Korkhaus y emulando a los clásicos gestómetros médicos. Resultados. Se obtuvieron dos tablas con valores desde los $19 \mathrm{~mm}$ hasta la medida del $30,5 \mathrm{~mm}$, medidas que corresponden a la sumatoria de los anchos mesiodistales de los incisivos inferiores. Los valores incluyen las proyecciones tanto para la maxila como para la mandíbula dadas por Moyers en el percentil 75, construyendo una "ruleta" con discos móviles giratorios, con valores para varones por un lado y para mujeres del otro lado. Conclusión. La ruleta es un instrumento que permite hallar los valores de los valores de Moyers para medir la discrepancia de arco en la dentición mixta con más facilidad yprecisión.

PALABRAS CLAVE: Ancho Mesiodistal Dental; Dentición Mixta; Ortodoncia; Modelos Lineales; Difusión De Innovación. (Fuente: DeCS BIREME)

\begin{abstract}
Objective. This study aimed to project the data from Moyers tables through linear regression equations and present a new instrument that facilitates the analysis of Moyers in mixed dentition. methods. The classical tables of Moyers were used through four linear equations projected data for both values, men and women, the roulette was built from a new table with values projected from the $19 \mathrm{~mm}$ to $30,5 \mathrm{~mm}$ corresponding to the sum of the lower incisors. Linear regression and linear equations were used to project values for both, maxilla and mandible. Roulette model was devised following the Korkhaus Orthometer model and emulating the classic medical gestation tables. Results. Two tables were obtained with new data values from $19 \mathrm{~mm}$ to $30,5 \mathrm{~mm}$ measurement, measures which correspond to the sum of the mesiodistal width of the lower incisors. The new values include projections for both, the maxilla and the mandible. Conclusion. The "roulette" is an instrument that permits find Moyers values for arch discrepancy measure in mixed dentition whith more precision and facility.
\end{abstract}

KEYWORDS: Dental Mesio Distal Widths; Mixed Dentition; Orthodontics; Linear Models; Diffusion of Innovation. (Source: Mesh NLM)

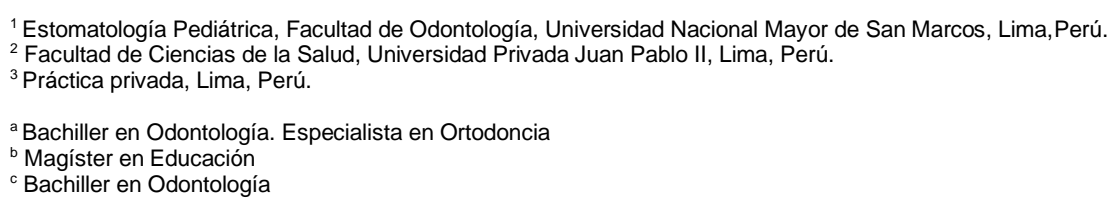

Citar como: Marengo H. Castro-Rodriguez Yuri. Bravo S. Calampa G. Torres L. La "ruleta de moyers", instrumento auxiliar para el análisis de la dentición mixta basado en la ecuación de regresión lineal por mínimos cuadrados. KIRU. 2020; enemar 17(1): 23-29. https://doi.org/10.24265/kiru.2020. v17n1.04 


\section{INTRODUCCIÓN}

Los problemas dentales, esqueléticos 0 ambos pueden generar maloclusiones ${ }^{(1)}$. El periodo de intercambio entre la dentición primaria a mixta y de mixta a permanente son de importancia e interés para el ortodoncista, debido a que el desarrollo de la dentición sufre cambios y la maloclusión puede mejorar o empeorar ${ }^{(2)}$. Estas pueden prevenirse en la etapa de la dentición mixta siempre que se logre detectar a tiempo y diagnosticar adecuadamente la patología(3).

En la etapa de la dentición mixta, es de suma importancia conocer a priori el tamaño de caninos y premolares que aún no han erupcionado y así determinar las diferencias de tamaño entre las arcadas y el ancho dental para poder encaminar el desarrollo de la oclusión del niño en crecimiento. Es frecuente, en ortodoncia, la falta de espacio, por lo que es necesario resolver este problema, ya que este manejo puede derivar principalmente en la prevención de apiñamientos ${ }^{(4)}$.

El análisis de Tanaka y Johnston permite predecir la erupción de los caninos y premolares permanentes, se basa en una ecuación de regresión lineal en el que a la sumatoria de los anchos mesiodistales de los incisivos inferiores divididos entre dos, se le suma $10,5 \mathrm{~mm}$ para predecir la suma del ancho de caninos y premolares inferiores y $11 \mathrm{~mm}$ para predecir la suma del ancho de caninos y premolares superiores. Al multiplicar por dos el resultado obtiene la suma del ancho de caninos y premolares no erupcionados de ambos lados del arco dentario(5).

Los esquemas predictores de Moyers y Tanaka y Johnston han logrado una amplia aceptación clínica; $\mathrm{y}$, esta última, una validez predictiva entre el espacio disponible y el espacio requerido en la planificación del tratamiento temprano, de ahí su importancia en la obtención de la precisión en su registro(6).

El análisis de Moyers es un método que se basa en tablas de percentiles para el maxilar y la mandíbula?. Este análisis mide los anchos mesiodistales de los dientes anteroinferiores y a partir de esa medida realiza predicciones del espacio (mediante la regresión lineal por mínimos cuadrados) para los caninos y premolares ${ }^{(8)}$. Este análisis fue creado para una población general y luego de 15 años de su publicación se desarrollaron tablas de percentiles para la población femenina y otra para la población masculina ${ }^{(9)}$. En muchas ocasiones, el manejo de los valores predictivos de la tabla de Moyers se dificulta, pues posee un límite de datos que se registran de $19,5 \mathrm{~mm}$ a $25,5 \mathrm{~mm}$, lo cual excluiría valores menores o mayores debido a piezas micro o macrodoncicas. Como no existe un estudio que cuantifique en una población peruana los casos de sumatoria de incisivos que sean mayores o menores a los presentados en las tablas, se justifica no la modificación, sino la ampliación de las tablas de Moyers al percentil 75. Sumado a esto, muchas veces las tablas son poco amigables para su manejo, sobre todo para aquellos estudiantes y/o profesionales que se inician en el estudio de la ortodoncia y el análisis de la dentición mixta.

El presente artículo tuvo como objetivo obtener los datos las ecuaciones de regresión lineal por mínimos cuadrados al $75 \%$, para determinar los valores por debajo y por encima de la Tabla de Moyers e idear un sistema que facilite el manejo de las predicciones clásicas hechas por Moyers y las nuevas medidas proyectadas a partir de la misma en un instrumento fácil de usar (la Ruleta de Moyers).

\section{MÉTODOS}

El presente estudio se realizó en las dependencias de la Facultad de Odontología de la Universidad Nacional Mayor de San Marcos, dentro del curso de Estomatología Integral del niño y del adolescente, para el concurso de mesas clínicas del año 2013.

Se utilizó la tabla clásica de Moyers tanto para la población masculina y femenina (Figura 1). A partir de la misma, se analizaron los datos que correspondían al percentil 75 por ser el más utilizado en el Perú, por sus usos y costumbres, al igual que en otras poblaciones en el mundo, por ejemplo, en la India ${ }^{(10)}$. Dichos datos fueron analizados mediante el método matemático de la regresión por mínimos cuadrados. Esta técnica de análisis numérico analiza un conjunto de pares ordenados: variable independiente, variable dependiente; y, mediante una familia de funciones, intenta encontrar la función continua que mejor se aproxime a los datos ${ }^{(11)}$. En nuestro caso, la variable independiente de nuestro par ordenado fue la medida de los anchos mesiodistales de los dientes anteroinferiores y la variable dependiente fue el valor predictivo que indica la tabla clásica de Moyers. A partir de la regresión, se logró obtener cuatro ecuaciones lineales para cada caso a analizar; así la primera fórmula para el sexo masculino: maxilar superior fue de $Y=10,5269+0,5 X$, para el maxilar inferior fue $Y=$ $11,8703+0,43 X$; para el femenino: maxilar superior fue $Y=14,7961+0,28 X$ y para el maxilar inferior $Y=8,839$ $+0,54 X$. A partir de cada ecuación, se proyectaron los datos para valores superiores al $25,5 \mathrm{~mm}$ y de esta forma se generaron valores predictivos mayores y menores a los de la tabla clásica de Moyers. 


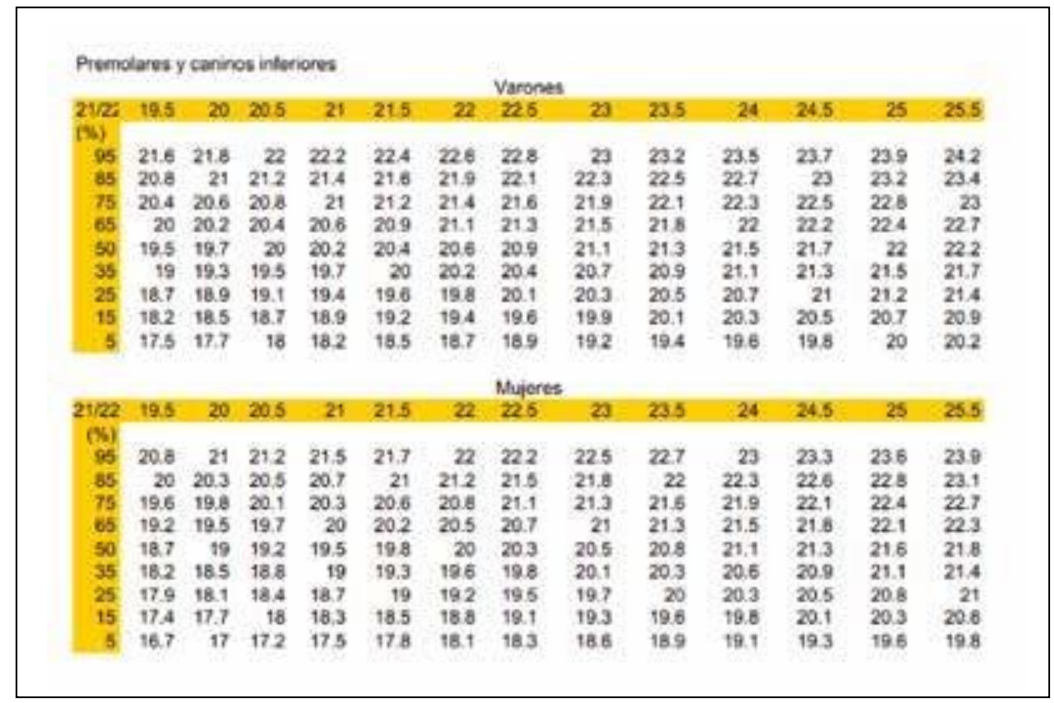

Figura 1. Proporción de docentes que contestaron correctamente las preguntas evaluadas en los centros escolares encuestados $(\%, I C)$.

Una vez obtenido los nuevos valores, se procedió a idear un sistema que permita unir la tabla clásica con los nuevos valores y se permita su fácil manejo al momento de analizar los modelos de dentición mixta. Nos basamos en el clásico Ortómetro de Korkhaus ${ }^{(12)}$, que consta de una ruleta giratoria con números impresos. Al girar el disco externo se tiene acceso a ventanas pudiéndose leer a través de ellas las medidas biométricas correspondientes a las dimensiones transversales y sagitales de ambas arcadas, luego de conseguir la suma de los incisivos
(Figura 2). Se confeccionó una ruleta que incluye los valores predictivos clásicos de Moyers y los nuevos datos proyectados utilizando como base el percentil 75 , los cuales se pueden visualizar en ventanas en la ruleta, ubicando la sumatoria de incisivos inferiores, aparecerán los valores de la predicción en dos ventanas una para el maxilar inferior y otra para el maxilar superior. La ruleta tiene dos caras: una para los valores del sexo masculino y otra para el sexo femenino.

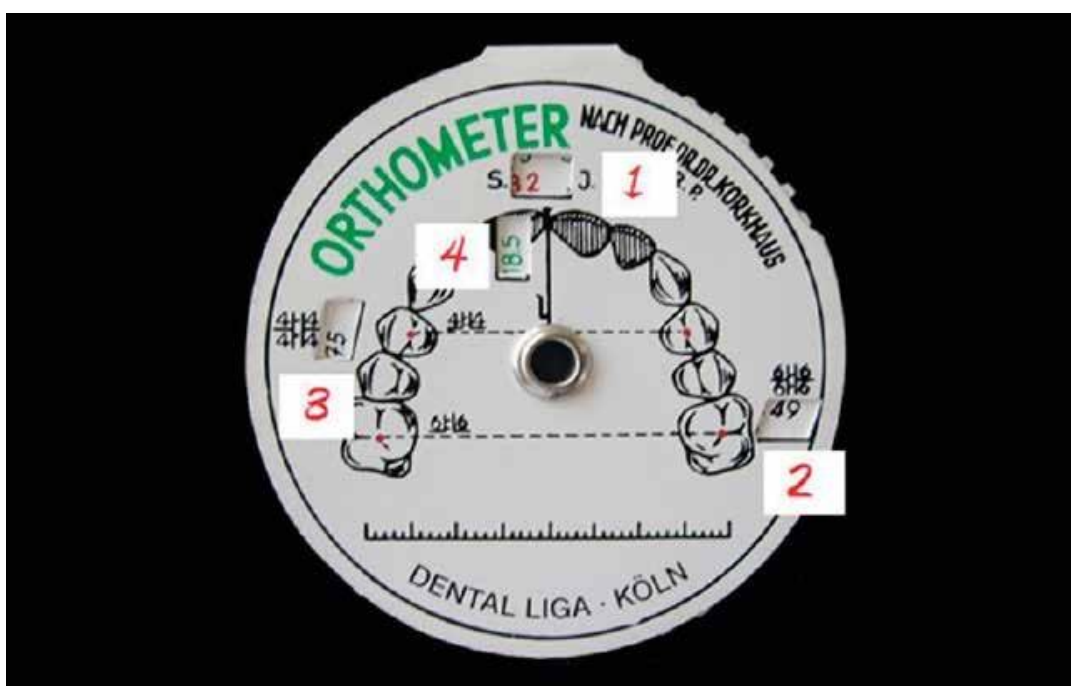

Figura 2. Ortómetro de Korkhaus, (1) indica la suma incisiva, (2) la dimensión transversal a nivel de la fosa mesial de los primeros molares, (3) la dimensión transversal a nivel de la fosa central de primeros premolares y (4) la longitud anterior, levantada desde de la dimensión transversal consignada en los premolares, perpendicular al centro de la papila de los incisivos centrales. 


\section{RESULTADOS}

Los datos proyectados a partir de la regresión por mínimos cuadrados fueron agrupados en dos tablas correspondientes a los datos predictivos para los caninos y premolares, tanto para el sexo masculino y para el sexo femenino para ambas arcadas (Tablas 1 y 2).

tabla 1. Valores proyectados con el percentil del $75 \%$ hasta los $30,5 \mathrm{~mm}$ para el sexo masculino.

\begin{tabular}{ccc}
\hline $\begin{array}{c}\text { Sumatoria } \\
\text { de incisivos } \\
\text { Inferiores } \\
\text { masculino }\end{array}$ & $\begin{array}{c}\text { Canino y } \\
\text { premolares } \\
\text { superiores }\end{array}$ & $\begin{array}{c}\text { Canino y } \\
\text { premolares } \\
\text { inferiores }\end{array}$ \\
\hline 26,0 & 23,5 & 23,2 \\
26,5 & 23,8 & 23,4 \\
27,0 & 24,0 & 23,6 \\
27,5 & 24,3 & 23,8 \\
28,0 & 24,5 & 24,1 \\
28,5 & 24,8 & 24,3 \\
29,0 & 25,0 & 24,5 \\
29,5 & 25,3 & 24,7 \\
30,0 & 25,5 & 24,9 \\
30,5 & 25,8 & 25,1 \\
\hline
\end{tabular}

tabla 2. Valores proyectados con el percentil del $75 \%$ hasta los $30,5 \mathrm{~mm}$ para el sexo femenino

\begin{tabular}{ccc}
$\begin{array}{c}\text { Sumatoria de } \\
\text { incisivos } \\
\text { Inferiores } \\
\text { femenino }\end{array}$ & $\begin{array}{c}\text { Canino y } \\
\text { premolares } \\
\text { superiores }\end{array}$ & $\begin{array}{c}\text { Canino y } \\
\text { premolares } \\
\text { inferiores }\end{array}$ \\
\hline 26,0 & 22,2 & 23,1 \\
26,5 & 22,3 & 23,4 \\
27,0 & 22,5 & 23,6 \\
27,5 & 22,6 & 23,9 \\
28,0 & 22,8 & 24,2 \\
28,5 & 22,8 & 24,5 \\
29,0 & 22,9 & 24,7 \\
29,5 & 23,1 & 25,0 \\
30,0 & 23,3 & 25,3 \\
30,5 & 23,5 & 25,6 \\
\hline
\end{tabular}

La ruleta fue confeccionada a partir de varios diseños preliminares hasta conseguir que todos los datos concuerden con sus respectivos valores predictivos, tanto para el maxilar y mandíbula, así como para el sexo masculino y femenino. Las portadas se realizaron con el manejo del diseño gráfico y el uso de imágenes y colores representativos para cada sexo. Los diseños definitivos se lograron a través de cuatro discos plastificados, dos para cada sexo. El disco superior posee la portada y los rótulos para cada análisis predictivo, el disco inferior contiene los datos predictivos tanto de la tabla clásica así como los nuevos valores proyectados (Figuras 3-5).
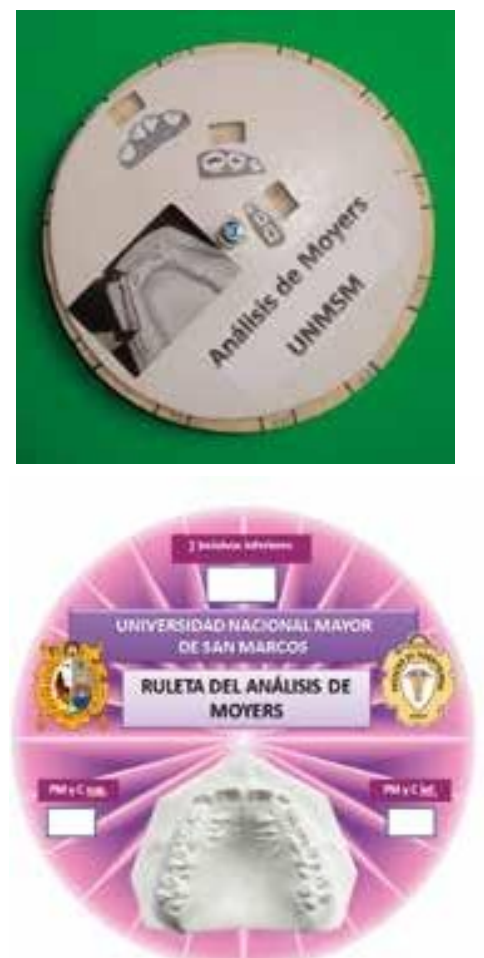

Figura 3. Primeros diseños de la ruleta para el análisis de Moyers.

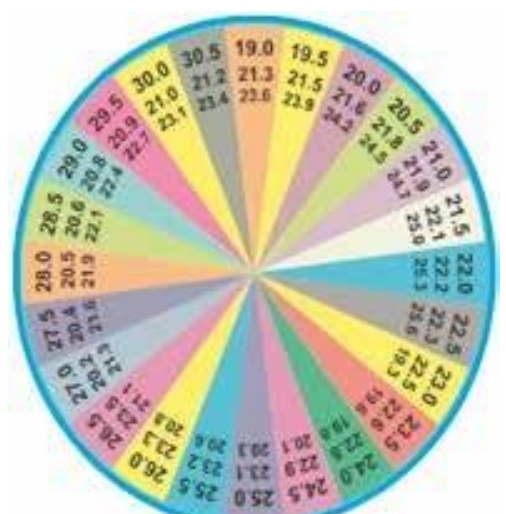

Figura 4. Disco interno con los datos predictivos de la tabla clásica y las nuevas tablas. 

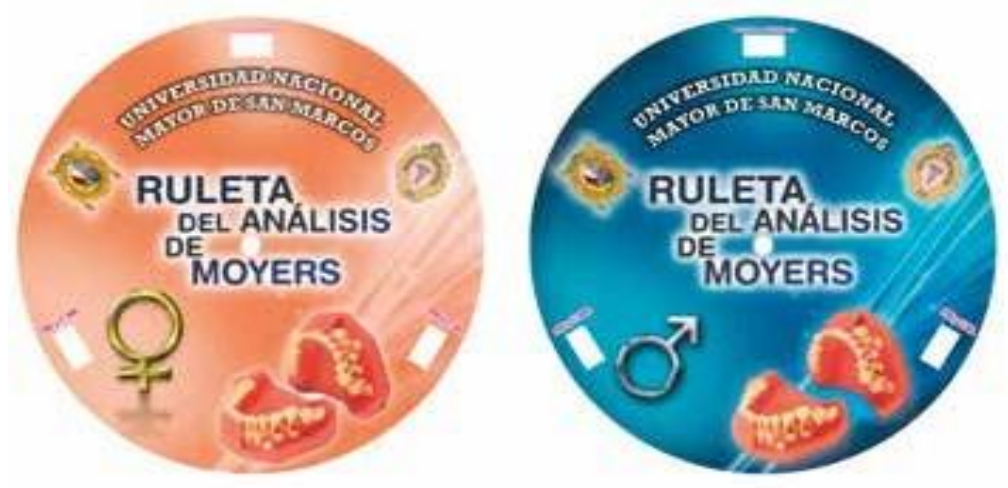

Figura 5. Disco externos con las portadas y los rótulos para cada análisis predictivo.

\section{DISCUSIÓN}

Dado que el presente estudio no posee antecedentes similares, en los que se idee un nuevo instrumento para facilitar el análisis de la dentición mixta de Moyers, la discusión se centra principalmente en la regresión que se utiliza para la elaboración de las tablas de predicción.

Uno de los análisis de la dentición mixta más popularizados es el de Moyers, este se basaba en las tablas que el autor proyectara para predecir el ancho mesiodistal de caninos y premolares ${ }^{(13)}$. Los porcentajes propuestos en estas tablas han sido determinados en una población norteamericana, siendo validados o modificados en diferentes partes del mundo ${ }^{14}$.

El análisis de espacio de la dentición mixta cuantifica el espacio disponible en el segmento lateral del arco para los caninos, los premolares y los ajustes oclusales necesarios. El análisis de la dentición mixta ayuda a valorar predictivamente la separación o apiñamiento futuro en el paciente, si todos los dientes primarios fueran cambiados por sus sucesores el día del análisis, no años más tarde. No cuantifica la disminución del perímetro que suele ocurrir durante el periodo transicional sin la pérdida de dientes ${ }^{(15,16)}$. Dicho análisis utiliza datos ya establecidos por el autor, además de percentiles que facilitan el trabajo estadístico a evaluar, Abu et al, concluyen que las Tablas de Probabilidad de Moyers son efectivas al $65 \%$ y $75 \%$ en los arcos superior e inferior de la población masculina y al $85 \%$ en la población femenina $^{(17)}$.

El presente estudio utilizó las tablas de probabilidad al $75 \%$ como percentil estándar y, a partir de la misma, se realizó una regresión matemática para calcular los valores de las medidas con datos que no aparecen en la tabla clásica de Moyers. El porqué de esta regresión radica en que en ciertas ocasiones las dimensiones tomadas en los modelos de estudio no son encontradas en la tabla clásica de Moyers, por lo que se imposibilita el análisis de la dentición mixta en estos tipos de casos. Cuando se puede obtener el valor correspondiente a la sumatoria de los anchos mesiodistales de caninos y premolares de manera predictiva, acertaremos en saber si el espacio disponible para estos es o no suficiente; y, si su alineación será la correcta. Se observa en la clínica de pregrado que muchas veces los estudiantes tienen problemas en hallar la predicción en las tablas, ya sea por error en la elección del percentil correcto, como por la falta del valor de la sumatoria de los incisivos inferiores en la tabla, intentando suplir esta falencia al ejecutar la predicción al tanteo ${ }^{(18)}$.

Varios estudios avalan la variación del ancho mesiodistal de los dientes según grupo étnico y el sexo ${ }^{(19-21)}$. Se hace necesario evaluar los análisis dentales probando su efectividad para poder realizar los ajustes necesarios para poderlos aplicar en nuestra población. Varios autores han reportado dimorfismo sexual en el tamaño dental de caninos y premolares, por lo que se realizaron análisis de dentición diferentes para hombres y mujeres. El problema de precisión de las tablas de Moyers puede disminuirse mientras se ajuste a cada población donde se aplica ${ }^{(22-24)}$.

Carbonell et al. ${ }^{(25)}$ en un análisis de modelos mediante el análisis de Moyers encontraron que los valores más comunes varían entre los $19 \mathrm{~mm}$ y los $26,5 \mathrm{~mm}$; sin embargo, acotan que en ciertos casos existen valores superiores que también deben ser evaluados. Nuestro estudio se basa en esos casos que escapan de los parámetros comunes. La regresión matemática es una técnica matemática que permite 
proyectar datos a través de figuras geométricas y mediante ecuaciones obtener nuevos valores, según un patrón lineal. Yuen et al. ${ }^{(26)}$ obtuvieroncoeficientes de relaciones lineales para incisivos inferiores, caninos y premolares, con lo cual obtuvieron cuatro ecuaciones de regresión lineal simple. Estas mismas cuatro ecuaciones fueron nuestra base para poder realizar la regresión de datos y, de esta manera, proyectarnos a datos superiores a los $26,5 \mathrm{~mm}$.

\section{CONCLUSIÓN}

Con el presente estudio se pretende presentar un nuevo instrumento que facilite el análisis de Moyers favoreciendo los procesos de enseñanza-aprendizaje de estudiantes de pre y posgrado de ortodoncia; asimismo, facilitar la comprensión del método y que además permita obtener valores fiables para medidas que no pertenezcan al estándar de la población. Recomendamos profundizar o ampliar los estudios para poder utilizar la Ruleta propuesta en un nivel clínico y obtener datos en la población peruana.

\section{Contribuciones de autoría:}

DSM, JPRV, AA, EV, VBB y MF diseñaron el estudio, recopilaron y analizaron los datos, redactaron y aprobaron el manuscrito.

\section{Fuente de financiamiento: Autofinanciado.}

Conflicto de intereses: Los autores declararon no tener conflictos de interés.

\section{REFERENCIAS BIBLIOGRÁFICAS}

1. Verzi P, Leonardi M, Palermo F. Mixed dentition space analysis in an Eastern Sicilian population. Minerva Stomatol. 2002;51(8):327-39.

2. Aquino C, Tirre M, Olivieria A. Applicability of three tooth size prediction methods for white Brazilians. The Angle Orthodontist. 2006;76(4):644-9

3. Mercado T. Nueva ecuación de regresión en una población de Lima-Perú. Tesis para optar el título de cirujano dentista. 2006. UNMSM.

4. Moyers E. Manual de Ortodoncia. 4ta ed. Buenos Aires. Editorial Médica Panamericana. 1992

5. Tanaka MM, Johnston LE. The Prediction of the size of unerupted canines and premolars in a contemporary orthodontic population. J Am Dent Assoc, 1974 Apr,88(4):798-801.

6. Brito FC, Nacif VC, Melgaço CA. Mandibular permanent first molars and incisisors as predictors of mandibular permanent canine and premolar widths: Applicability and consistency of the method. Am J Orthod Dentofacial Orthop. 2014 Mar;145(39:393-8.

7. William B, Annet K, Louis M, Mugisha C .Prediction of width of un-erupted incisors, canines and premolars in a Ugandan population: A cross sectional study. BMC Oral Health. 2012,12:23 2-6.

8. Jaroontham J, Godfrey K. Mixed dentition space analysis in a Thai population. Eur $\mathrm{J}$ Orthod. 2000; 22:127-134.

9. Diagne F, Diop-Ba K, Ngom P, Mbow K: Mixed dentition analysis in a Senegalese population: elaboration of prediction tables. Am J Orthod Dentofac Orthop. 2003;124:178-183.

10. Mittar M, Dua VS, Wilson S. Reliability of permanent mandibular first molars and incisors widths as predictor for the width of permanent mandibular and maxillary canines and premolars. Contemp Clin Dent. 2012;3:812

11. Dawson B, R, Trapp. Bioestadística médica. 4ta. Ed. México: Ed. El Manual Moderno, 2005

12 Leiva N, Cauvi D, Beltrán S, Barrios U. Comparación en modelos de estudio de la longitud anterior del maxilar superior de niños con hendidura labial unilateral y fisura velopalatina operados con respecto a la medida determinada por Korkhaus. Acta odontológica venezolana. 2012;50(4):1-8.

13. Jaiswal AK, Paudel KR, Shrestha SL, Jaiswal S. Prediction of space availablefor unerupted permanent canine and premolars in a Nepalese population. $J$ Orthod. 2009;36:253-9.

14. Boboc A, Dibbets J. Prediction of the mesiodistal width of unerupted permanent canines and premolars: a statistical approach. Am J Orthod Dentofacial Orthop. 2010;137:503-7.

15. Talebi M, Parisay I, Sarraf A, Mazhari F. Regression equations for predicting the size of unerupted canines and premolars in an Iranian population: a pilot study. J Contemp Dent Pract. 2010;11:33-40.

16. Cattaneo C, Butti AC, Bernini S, Biagi R, Salvato A. Comparative evaluation of the group of teeth with the best prediction value in the mixed dentition analysis. Eur J Paediatr Dent. 2010;11:23-6.

17. Abu ES, Qudeimat MA. Mixed dentition space analysis in a Jordanian population: comparison of two methods. Jordan Int J Paediatr Dent. 2006; 16(2):10410.

18. Carrillo JJP, Rubial MC, Albornoz C, Villalba S, Damiani P, de Cravero MR. Applicability of the Moyers' Probability Tables in Adolescents with Different Facial Biotypes. Open Dent J. 2017; 26(11):213-220.

19. Grover N, Saha S, Tripathi AM, Jaiswal JN, Palit M. Applicability of different mixed dentition analysis in Lucknow population. J Indian Soc Pedod Prev Dent. 2017;35(1):68-74.

20. Bhatnagar A, Sinha AA, Chaudhary S, Manuja N, Kaur $\mathrm{H}$, Chaitra TR. Accuracy and evaluation of a new regression equation in predicting the width of unerupted permanent canines and premolar teeth. Eur Arch Paediatr Dent. 2017;18(1):31-37.

21. Gyawali R, Shrestha BK, Yadav R. Mixed dentition space analysis among Nepalese Brahmins/Chhetris. BMC Oral Health. 2016;17(1):36.

22 Shobha MB, Ajs S, Manoj K, Srideevi E, Sridhar M, Pratap G. Applicability of Two Universally Accepted Mixed Dentition Analysis on a Sample from Southeastern Region of Andhra Pradesh, India. Ann Med Health Sci Res. 2016;6(3):176-80.

२3. Shahid F, Alam MK, Khamis MF. New prediction equations for the estimation of maxillary mandibular canine and premolar widths from mandibular incisors and mandibular first permanent molar widths: A digital model study. Korean J Orthod. 2016;46(3):171-9.

24. Al-Dlaigan $\mathrm{YH}$, Alqahtani ND, Almoammar $\mathrm{K}$, AlJewair T, Salamah FB, Alswilem M, Albarakati SF. Validity of Moyers mixed dentition analysis for Saudi population. Pak J Med Sci. 2015;31(6):1399-404.

25. Carbonell O, Gonzáles G, Céspedes R. Estudio 
comparativo entre la tabla de Moyers nivel del $50 \%$ y los valores predictivos de Tanaka y Jhonston Rev Cubana Ortod. 1999;14(1):18-21.

26. Yuen KK, Tang EL, So LL. Mixed dentition analysis for Hong Kong Chinese. Angle Orthod. 1998;68(1):21- 